\title{
Anatomia comparada, histoquímica e fitoquímica dos órgãos vegetativos de espécies do gênero Ocimum L. (Lamiaceae)
}

A descoberta das propriedades terapêuticas das plantas é algo antigo, mas para sua utilização, precisa-se identificar as espécies corretamente, e muitas vezes, caracteres morfológicos não são o suficiente. Portanto, o objetivo deste trabalho foi diferenciar através da anatomia, histoquímica e fitoquímica três espécies do gênero Ocimum, amplamente utilizadas para produção de remédios naturais. Para tanto, foram coletadas Ocimum basilicum, 0 . gratissimum e Ocimum campechianum em quintais urbanos no município de Alta Floresta, estado de Mato Grosso, Brasil. Anatomicamente as espécies Ocimum basilicum e O. gratissimum são similares, enquanto a diferença de 0 . campechianum se dá pelo número de feixes vasculares e estrutura de raiz. Os testes histoquímicos mostraram a semelhança nos compostos produzidos de potencial medicinal, assim como a fitoquímica revelou a presença de taninos, flavonoides e alcaloides, compostos antioxidantes, expectorantes e anestésicos. A partir dos resultados obtidos, podemos concluir que as espécies possuem anatomia similar assim como a presença de compostos secundários, os quais são tradicionalmente indicados para uso medicinal.

Palavras-chave: Plantas Medicinais; Metabólitos Secundários; Alfavaca.

\section{Comparative anatomy, histochemistry and phytochemistry of the vegetative organs of species of the genus Ocimum L. (Lamiaceae)}

\begin{abstract}
The discovery of the therapeutic properties of plants is something old, but for its use, it is necessary to identify the species correctly, and many times, morphological characters are not enough. Therefore, the objective of this work was to differentiate anatomically, histochemically and phytochemically three species widely used for the production of natural remedies of the genus Ocimum. Therefore, to carry out the laboratory analyzes, Ocimum basilicum, $\mathrm{O}$. gratissimum and Ocimum campechianum were collected in urban gardens in the municipality of Alta Floresta, state of Mato Grosso, Brazil. Anatomically the species Ocimum basilicum and $\mathrm{O}$. gratissimum are similar, while the difference of $\mathrm{O}$. campechianum is due to the number of vascular bundles and root structure. Histochemical tests shown the similarity in compounds produced with medicinal potential and phytochemistry revealed the presence of tannins, flavonoids and alkaloids, antioxidant compounds, expectorants and anesthetics. From the results obtained, we can conclude that the species have similar anatomy as well as the presence of secondary compounds, which are traditionally indicated for medicinal use.
\end{abstract}

Keywords: Medicinal Plants; Secondary Metabolites; Alfavaca.

Topic: Desenvolvimento, Sustentabilidade e Meio Ambiente

Reviewed anonymously in the process of blind peer
Received: 09/02/2020

Approved: 07/03/2020
Vera Lúcia Pegorini Rocha (iD

Universidade do Estado de Mato Grosso, Brasil http://lattes.cnpq.br/4825442790338328

http://orcid.org/0000-0003-0539-948X verapegorinirocha@gmail.com

\section{Odair de Souza Fagundes}

Universidade do Estado de Mato Grosso, Brasil odair.bio.afmt@gmail.com

Virgilia Aparecida Pegorini Rocha

Universidade do Estado de Mato Grosso, Brasil http://lattes.cnpq.br/1105523955066811 virgilia_pegorini@hotmail.com

\author{
Ricardo Eduardo Vicente (iD \\ Universidade do Estado de Mato Grosso, Brasil \\ http://lattes.cnpq.br/3136128865901159 \\ http://orcid.org/0000-0003-2640-2537 \\ ricardomyrmex@gmail.com \\ Norberto Gomes Ribeiro Junior \\ Universidade do Estado de Mato Grosso, Brasil \\ http://lattes.cnpq.br/0708897518142620 \\ http://orcid.org/0000-0002-7518-7952 \\ norberto.gomes@unemat.br \\ Oscar Mitsuo Yamashita (it) \\ Universidade do Estado de Mato Grosso, Brasil \\ http://lattes.cnpq.br/1761470950121024 \\ http://orcid.org/0000-0001-6715-626X \\ yama@unemat.br
}

Ivone Vieira da Silva (iD

Universidade do Estado de Mato Grosso, Brasil http://lattes.cnpq.br/4828497736925374 http://orcid.org/0000-0003-0281-0608 ivibot@hotmail.com
Referencing this:

ROCHA, V. L. P.; FAGUNDES, O. S.; ROCHA, V. A. P.; VICENTE, R. E.; RIBEIRO JUNIOR, N. G.; YAMASHITA, O. M.; SILVA, I. V.. Anatomia comparada, histoquímica e fitoquímica dos órgãos vegetativos de espécies do gênero Ocimum L. (Lamiaceae). Revista Ibero Americana de Ciências Ambientais, v.11, n.2, p.266-277, 2020. DOI: http://doi.org/10.6008/CBPC2179-6858.2020.002.0027 


\section{INTRODUÇÃO}

A descoberta das propriedades terapêuticas das plantas se deu quando o homem passou a observar que alguns animais quando adoentados se alimentavam de certas ervas ou arbustos. Essas informações foram repassadas ao longo de gerações (muitas das vezes associadas a mitos e credos religiosos), o que formou parte importante da cultura de certas regiões (JORGE, 2013). O uso de plantas medicinais pelos seres humanos tem sido objeto de estudo de vários levantamentos etnobotânicos e estes, quando aliados a técnicas como a histoquímica podem trazer resultados importantes uma vez que, ao localizar os princípios ativos presentes nas plantas, é possível atribuir ou não uma indicação terapêutica a elas (SILVA et al., 2014). A padronização para o uso de plantas medicinais teve início na década de 70 na Alemanha, com um marcador químico ou pelo próprio princípio ativo, para garantir que o paciente que tomasse um medicamento fitoterápico e tivesse a certeza de que naquele comprimido ou cápsula continha a quantidade reprodutível e necessária para fazer o efeito terapêutico (SIMON, 2011).

A família Lamiaceae possui muitas espécies medicinais, sendo constituída por 7.886 espécies distribuídas em 245 gêneros em regiões tropical e subtropical (HARLEY et al., 2015). No Brasil a família é representada por 7.203 espécies e 236 gêneros (STEVENS, 2018). Entre os gêneros medicinais cultivados no Brasil, Ocimum L. merece destaque pelo uso de suas folhas nas práticas da medicina caseira e na culinária. Muitos integrantes desse gênero apresentam propriedades como a presença de óleo essencial, que é secretado por tricomas glandulares e que são de grande valor adaptativo e taxonômico em estudos botânicos (RIVA et al., 2014).

A anatomia vegetal é uma ferramenta de grande importância na compreensão botânica, pois compreende estudos da morfologia externa e interna da planta, e se apresenta como ferramenta de controle de qualidade à uma planta utilizada na indústria de medicamentos e condimentos (ESTEVES, 2011). A anatomia possibilita, em princípio, a diferenciação entre espécies, principalmente quando estas espécies são aparentemente semelhantes ou botanicamente próximas. A grande variedade, além da ampla ocorrência de Ocimum associada à interferência do homem no seu cultivo e da hibridação do gênero, tornam sua identificação complexa (FURLAN et al., 2012). Nesse sentido, objetivamos descrever e comparar a anatomia dos órgãos vegetativos de Ocimum basilicum (Willd) Benth. var pilosium, Ocimum campechianum Mill e Ocimum gratissimum L., a fim de diferenciá-las anatomicamente, histoquimicamente e fitoquimicamente e, também, a partir dos órgãos indicados como medicinais, verificar a presença de estruturas secretoras e identificar metabólitos secundários, contribuindo com dados para a medicina popular e futuros estudos farmacobotânicos.

\section{MATERIAIS E MÉTODOS}

Coletamos o material vegetal (folha, pecíolo, caule e raiz) das três espécies de Ocimum em quintais urbanos no município de Alta Floresta, estado de Mato Grosso, Brasil e analisamos no Laboratório de Biologia Vegetal da Universidade do Estado de Mato Grosso, Campus de Alta Floresta. Depositamos o material 
botânico fértil de Ocimum basilicum (Willd) Benth. var pilosium, Ocimum campechianum Mill e Ocimum gratissimum L., no Herbário da Amazônia Meridional (HERBAM), com identificação botânica confirmada e registradas sob o voucher $14.000,13.172$ e 13.171 .

Para a análise de similaridade, realizamos a junção dos dados de presença (1) e ausência (0) dos caracteres anatômicos e histoquímicos das três espécies em uma análise de agrupamento para 80 variáveis. Na realização da matriz de similaridade empregamos os métodos UPGMA (Unweighted Pair Group Method using Arithmetic Averages) e WPGMA (Weighted Pair group Method with Averaging) através do programa estatístico (R) utilizando a função vegdist do pacote vegan (OKSANEN et al., 2013), e utilizamos o método com maior coeficiente de correlação cofenético (CCC), no caso UPGMA sobre a matriz de similaridade de Jaccard. Geramos um dendrograma a partir da função Hclust do pacote cluster (MAECHLER et al., 2017), agrupando espécies com maior similaridade em relação as características anatômicas e histoquímicas.

Para a análise da epiderme foliar utilizamos o método de dissociação de Jeffrey (KRAUS et al., 1997) modificado, onde acondicionamos porções foliares em solução de água oxigenada (Volume 30) e ácido acético glacial na proporção de 1:1 e mantivemos em estufa a $65^{\circ} \mathrm{C}$ por um período. Cada espécie apresentou um período específico de reação, variando de 24 a 72 horas. Após este período, lavamos as amostras em água destilada e, com auxílio de um pincel, separamos as duas superfícies epidérmicas, que posteriormente coramos com fucsina básica (ROESER, 1962) e montamos as lâminas. Registramos as imagens por meio de fotomicroscópio Leica ICC50 acoplado a um computador com o software LAZ EZ versão 1.7.0.

Para as análises histoquímicas utilizamos seç̧ões histológicas do material fresco (folha, pecíolo, caule e raiz) obtidas com cortes à mão livre com auxílio de lâmina de aço e submetemos aos testes: Sudão IV para lipídeos totais (PEARCE, 1980), Reagente de NADI para óleos essenciais (DAVID et al.DE, 1964), Vanilina clorídrica para taninos (MACE et al., 1974), Cloreto de ferro III para compostos fenólicos (JOHANSEN, 1940), Dragendorff para alcaloides (SVENDSEN et al., 1983), Lugol (IKI) para amido (JOHANSEN, 1940), Ácido tânico e Cloreto de ferro III a 3\% para mucilagens (PIZZOLATO et al., 1973), 2,4 Dinitrofenilhidrazina para terpenoides (GANTER et al., 1970), Ácido Sulfurico para lactonas sesquiterpénicas (GEISSMAN et al., 1971) e Tricloreto de antimônio para esteroides (HARDMAN et al., 1972). Relaizamos testes fitoquímicos para detecção de flavonoides, taninos, saponina e alcaloides por meio de extratos alcoólicos, para isso foi necessário a transformação das partes vegetativas em pó.

\section{RESULTADOS E DISCUSSÃO}

Com o CCC de 0,997, o método de agrupamento UPGMA nos revelou maior semelhança entre as espécies Ocimum basilicum e Ocimum gratissimum (Fig. 1). Em vista frontal, as três espécies estudadas apresentam epiderme foliar com paredes sinuosas em ambas as faces (Figs. 2A-2F), sendo a sinuosidade mais acentuada na face abaxial. As folhas são anfiestomáticas, com estômatos diacíticos, mais frequentes na face abaxial (Figs. 2D-2F). 


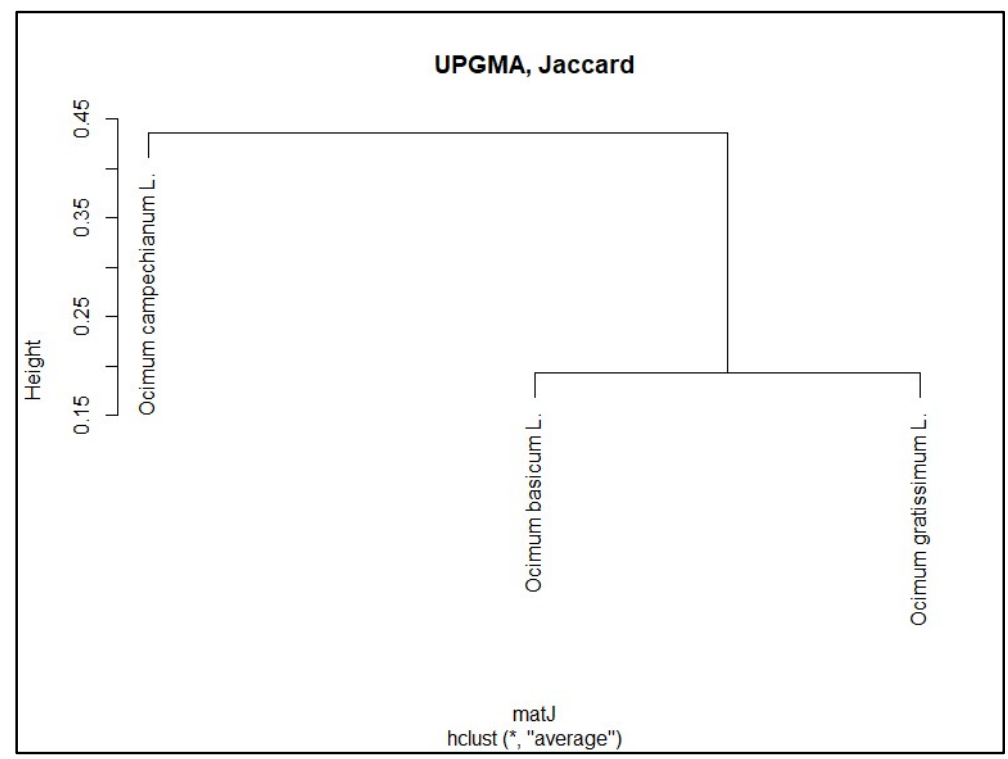

Figura 1: Dendograma construído a partir de 80 caracteres anatômicos e histoquímicos dos órgãos vegetativos com as espécies de Ocimum basilicum, Ocimum campechianum e Ocimum gratissimum.

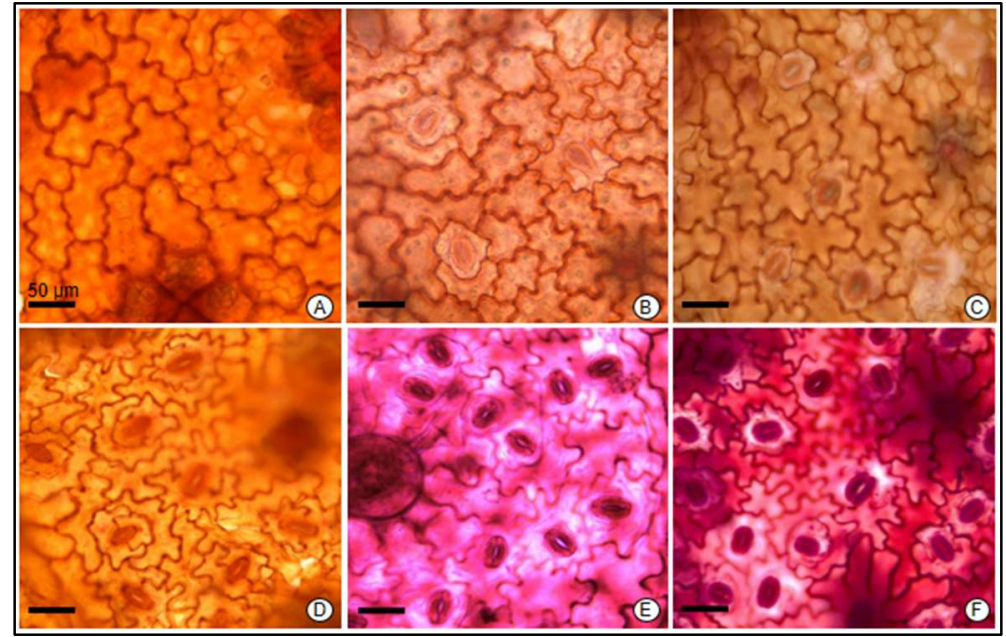

Figura 2: Secções paradérmicas da lâmina foliar evidenciando as células epidérmicas adaxiais $(A, B$ e $C)$ e abaxiais (D, E e F) com paredes sinuosas em $\mathrm{O}$. basilicum, $\mathrm{O}$. campechianum e $\mathrm{O}$. gratissimum respectivamente.

Em vista transversal, na região da nervura central, as espécies apresentam de três a cinco camadas de colênquima angular e de quatro a seis camadas de parênquima de preenchimento voltadas para ambas as faces (Figs. 3A-3C). Furlan et al. (2012) observaram organização anatômica semelhante para Ocimum basilicum. Os feixes vasculares são do tipo colateral aberto raso. Ocimum basilicum e 0 . gratissimum apresentam uma divisão dos arcos contendo os feixes vasculares na região central do mesofilo (Figs. 3A e 3C). $O$. gratissimum e $O$. basilicum possuem quatro feixes acessórios, enquanto $O$. campechianum apenas dois (Figs. 3A-3C), sendo evidente a diferença dos números de feixes. Características semelhantes foram encontradas por Oliveira et al. (2013), para outra espécie da família Lamiaceae, Scutellaria agrestis A. St. Hil. ex Benth, que apresentam sistema vascular colateral, dispostos em três feixes vasculares sendo um central, maior, com arco aberto, e dois auxiliares, cilíndricos, menores.

A epiderme das três espécies é uniestratificada, constituída por células ovaladas a retangulares, alongadas periclinalmente e revestida por cutícula delgada (Figs. 3D-3F), caracteres já descrito para $O$. gratissimum (SIMON et al., 2011; COSTA et al., 2014). 
A lâmina foliar das três espécies estudadas apresentam por toda sua extensão, ondulações pronunciadas, onde registramos a presença de estômatos e tricomas glandulares peltados (Fig. 3D). Observamos a presença de câmaras subestomáticas volumosas com estômatos no mesmo nível das demais células epidérmicas abaxiais (Figs. 3E e 3F). As espécies são dorsiventrais, composta por parênquima paliçádico constituído por uma camada e parênquima lacunoso com uma média de quatro camadas com arranjo aleatório e muitos espaços intercelulares (Fig. 3D-3F). A disposição dos tecidos do mesofilo foliar das três espécies é anatomicamente similar. Tais características anatômicas também foram evidenciadas por diferentes autores para espécies do gênero Ocimum (FURLAN et al., 2012; RIVA et al., 2014; ROCHA et al., 2015).

No pecíolo de $O$. basilicum, $O$. campechianum e $O$. gratissimum, em corte transversal observamos inúmeros tricomas tectores pluricelulares e tricomas glandulares peltados, sendo estes mais evidentes na face adaxial (Figs. 3G-3I). O mesmo foi observado no pecíolo em outras espécies do gênero (CANINI, 2013), que possuem tricomas tectores e dois tipos de tricomas glandulares, capitados e peltados, sendo os tricomas tectores mais frequentes na face adaxial. Assim como na nervura principal da lâmina foliar, a epiderme é similar a descrita para a lâmina foliar e apresenta, logo abaixo da epiderme, até quatro camadas de colênquima que formam uma faixa contínua seguida de parênquima fundamental (Figs. 3G-3I), resultados semelhantes foram descritos por Canini (2013), onde Ocimum apresentam de uma a três camadas de colênquima e três a seis camadas de células de parênquima de preenchimento.

Ainda no pecíolo, em $O$. basilicum e $O$. gratissimum, o feixe vascular principal é do tipo colateral em arco aberto, formado por sete feixes, sendo dois maiores centrais, três acessórios deslocados voltados para face adaxial e outros dois acessórios nas suaves projeções das extremidades voltados para a face adaxial (Figs. 3G e 3I), sendo confirmado por Rocha et al. (2015) em O. basilicum. Já em O. campechianum, o feixe vascular principal é do tipo colateral em arco raso aberto, formado por três feixes, sendo dois acessórios voltado para a face adaxial (Fig. 3H). A região cortical da estrutura, nas três espécies, é preenchida por parênquima de preenchimento com células isodiamétricas (Figs. 3G-3I). O que difere o pecíolo das três espécies são o número de feixes e a disposição dos mesmos. Tais características são muito semelhantes com os resultados de Costa et al. (2014), onde descrevem o pecíolo de $O$. gratissimum com feixe vascular principal do tipo colateral, em arco raso aberto, constituído por cinco feixes, e um menor em cada uma das projeções laterais do pecíolo. Segundo Metcalfe et al. (1979), a estrutura vascular do pecíolo é também de importância taxonômica para a identificação do gênero.

Através das secções transversais do caule constatamos que as espécies apenas se diferenciam em sua morfologia externa. As três espécies estudadas apresentam no caule contorno quadrangular (Figs. 4A4C), com faces levemente convexas revestidas por epiderme uniestratificada (Figs. 4F) e com os mesmos tipos de tricomas observados na lâmina foliar e no pecíolo (Figs. 4D e 4E). Entretanto apenas $O$. campechianum possui no formato quadrangular reentrâncias. $O$ colênquima é angular e se concentra nos ângulos do caule, com seis a oito camadas (Fig. 4F). O feixe vascular é do tipo colateral em arco fechado e calotas de fibras associadas ao floema (Fig. 4A, B, C e 4F). O córtex é preenchido pelo parênquima medular e 
não registramos estruturas secretoras (Fig. 4A, B, C e 4F). Característica de estruturação anatômica do caule semelhantes foram observadas no gênero Ocimum por Duarte et al. $(2005 ; 2007)$, Simon et al. (2011) e Costa et al. (2014).

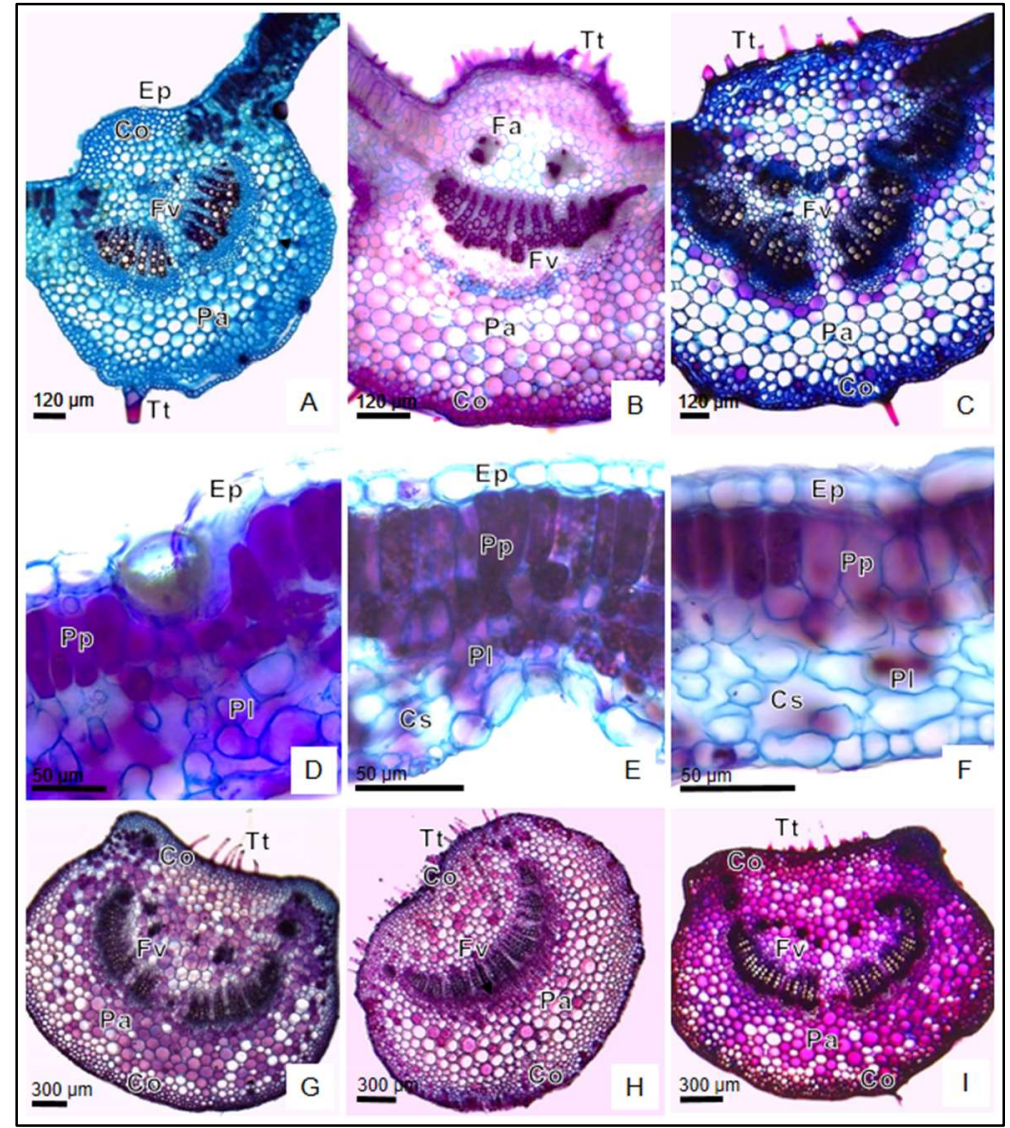

Figura 3: Secções transversais foliares de $O$. basilicum (A, D e G), O. campechianum (B, E e H) e $O$. gratissimum (C, F e I): Epiderme (Ep), Colênquima (Co), Feixes acessórios (Fa), Feixes vasculares ( $F v)$, Parênquima ( $\mathrm{Pa}$ ), Parênquima lacunoso (PI), Parênquima paliçádico $(\mathrm{Pp})$ e Tricoma tector $(\mathrm{Tt})$.

As três espécies descritas possuem muitas similaridades em secções transversais da raiz (Figs. 4G-4I), apenas alguns caracteres são diferenciados como: 0 . basilicum e 0 . campechianum que apresentam quatro camadas de felogênio, enquanto 0 . gratissimum possui sete camadas (Fig. 4I). O. basilicum e O. gratissimum apresentam nesse tecido um sistema bem desenvolvido de espaços intercelulares, os chamados lobos de conexão com paredes lignificadas (Fig. 4J e 4L), similar ao de Scutellaria agrestis também da família Lamiaceae (OLIVEIRA, 2011). O súber ainda está em diferenciação na região mediana do órgão. A feloderme se apresenta com duas camadas nas três espécies (Fig. 4K) e seis camadas de raios parenquimáticos de células tabulares envolvendo os feixes vasculares em raios (Fig. 4H e 4I).

Através dos testes histoquímicos identificamos a presença de compostos secundários nas espécies estudadas, havendo maior similaridade entre as espécies Ocimum basilicum e O. gratissimum. Há um crescente interesse em identificar compostos em plantas que possam ser consumidos como alimento pelos indivíduos, como parte habitual de suas dietas e tratamentos medicinais. Entretanto, o uso de plantas destinadas a medicina popular tem sido feito, sem um conhecimento dos compostos nelas existentes. Esses compostos ocorrem em diversas plantas, mas somente algumas espécies possuem aplicação terapêutica (JORGE, 2013). Das substâncias químicas evidenciadas com os testes histoquímicos em $O$. basilicum, $O$. 
campechianum e O. gratissimum, foram detectadas nas três espécies: amido, compostos fenólicos, lipídios totais, mucilagens e óleos essenciais.

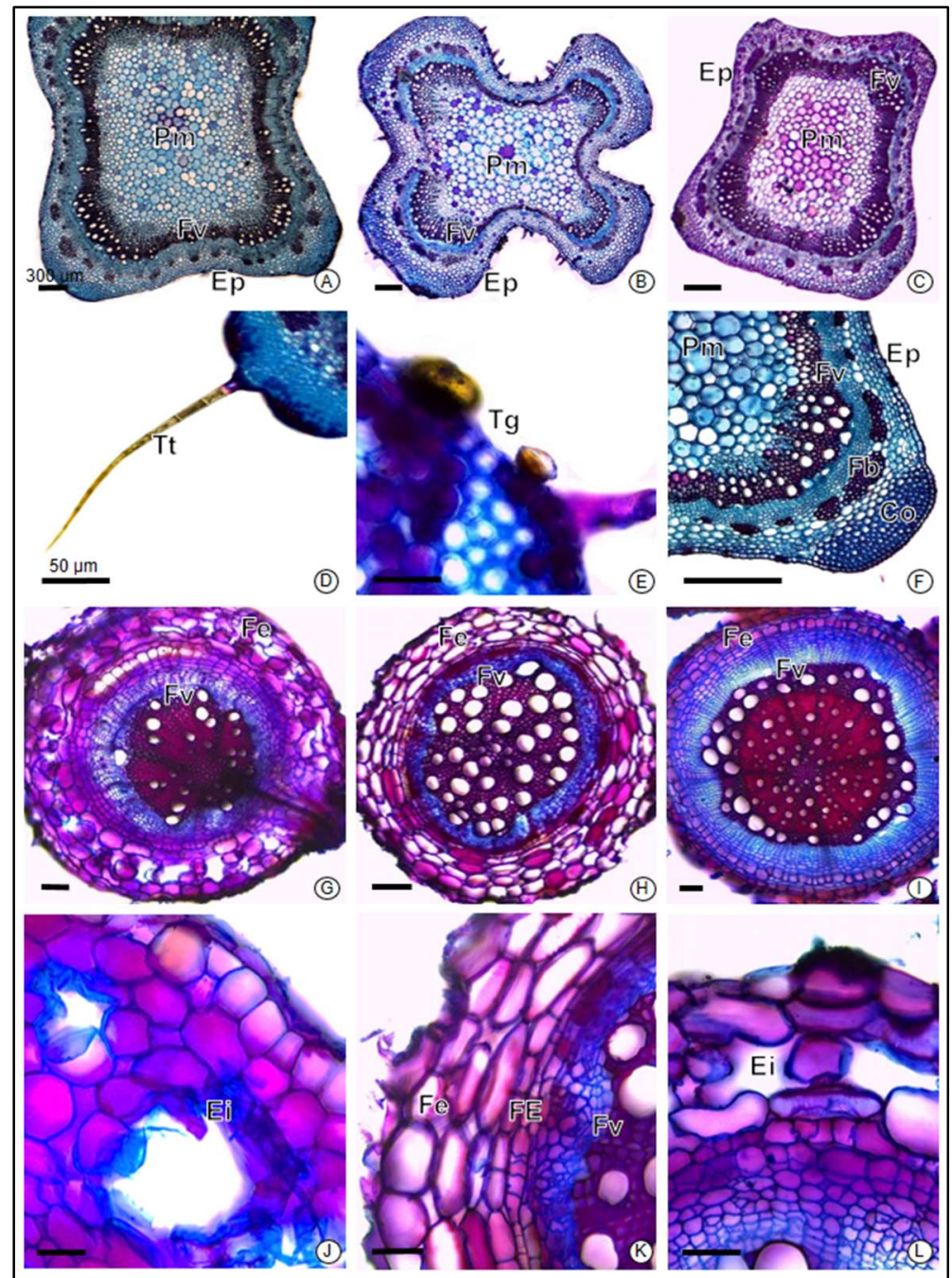

Figura 4: Secções transversais do caule e raiz de $O$. basilicum (A, D, G e J), $O$. campechianum (B, E, H e K) e $O$. gratissimum (C, F, I e L). Colênquima (Co), Epiderme (Ep), Espaços intercelulares (Ei), Feixes vasculares (Fv), Feloderma (FE), Felogênio (Fe), Fibras (Fb), Parênquima medular (Pm), Tricoma tector (Tt) e glandular (Tg).

Observamos nas seç̧ões transversais dos órgãos vegetativos de $O$. basilicum submetidas aos testes histoquímicos a presença de amido (Fig. 5A) em todos os órgãos da planta. Os compostos fenólicos (Fig. 5B) concentram-se nos tricomas da nervura central, no colênquima e epiderme adaxial do pecíolo, e no colênquima caulinar. Os lipídios totais (Figs. 5C e 5D) foram encontradas no parênquima paliçádico e no tricoma caulinar. Também encontramos mucilagens (Fig. 5E) em todos os órgãos da planta e óleos essenciais (Figs. 5F-H) encontramos nos tricomas da nervura central, no colênquima e epiderme adaxial do pecíolo, e no colênquima caulinar.

Seç̧ões transversais dos órgãos vegetativos de $O$. campechianum evidenciaram a presença de amido (Figs. $5 \mathrm{l}$ e 5J) em três dos quatro órgãos analisados, sendo, na epiderme da nervura central, no parênquima lacunoso na face abaxial do mesofilo, no parênquima angular da face adaxial do pecíolo e no colênquima angular no ângulo do caule. Os compostos fenólicos foram encontrados no parênquima angular do pecíolo (Fig. 5K). Os lipídios totais foram encontrados no tricoma da nervura central e no colênquima do pecíolo e 
do caule (Figs. 5L e 5M). Mucilagens (Fig. 5N) concentram-se no parênquima cortical do caule e no sistema vascular da raiz e óleos essenciais (Figs. 50 e 5P) no parênquima da nervura central, no parênquima do mesofilo, no colênquima angular do pecíolo e no parênquima medular do caule.

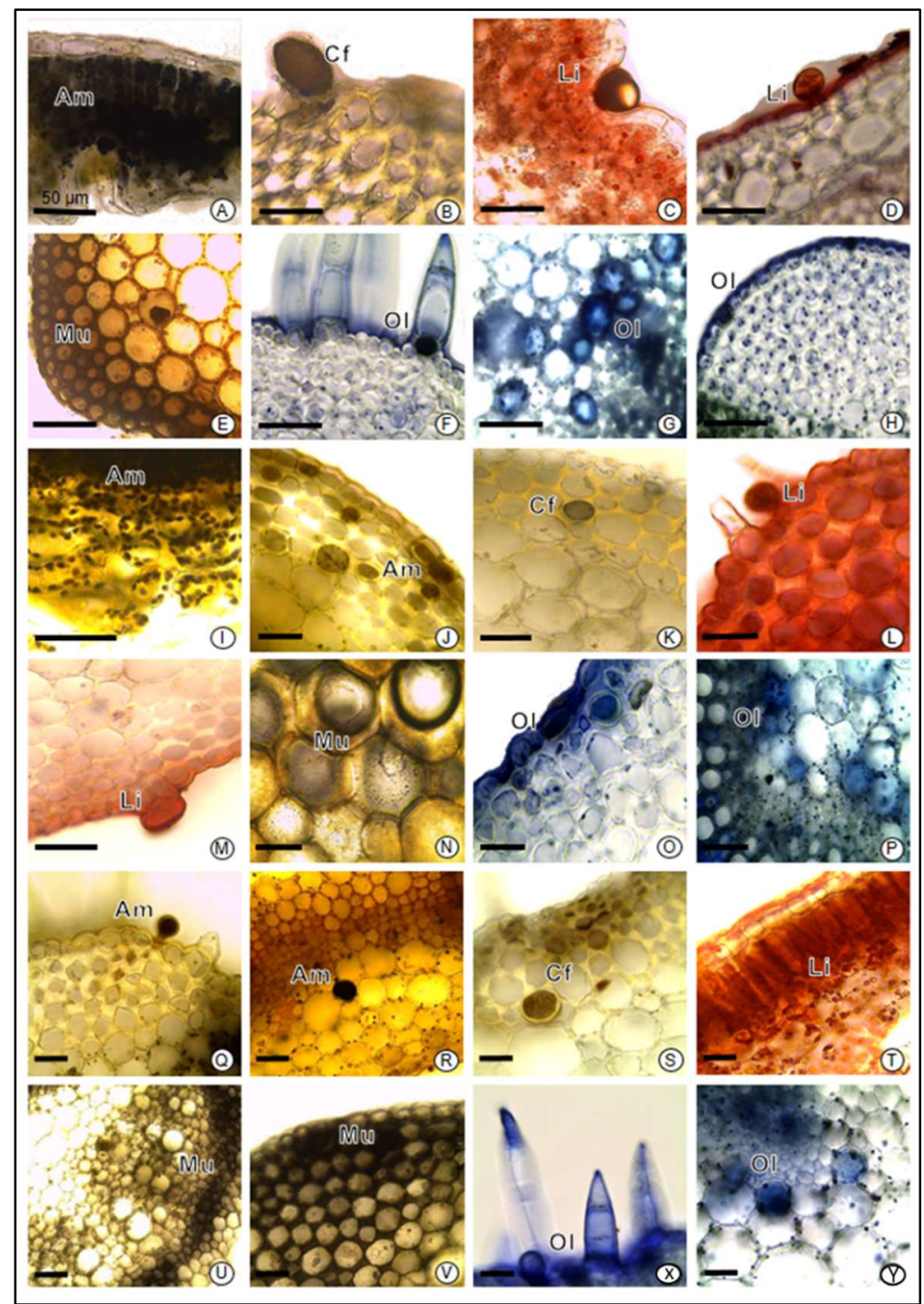

Figura 5: Secções transversais dos órgãos vegetativos de $O$. Basilicum (A-H), O. campechianum (I-P) e $O$. Gratissimum (Q-Y) submetidas aos testes histoquímicos, evidenciando a presença de amido (Am), compostos fenólicos (Cf), lipídios totais (Li), mucilagens (Mu), e óleos essenciais (OI).

Observamos nos testes histoquímicos realizados em 0 . gratissimum resultados positivos para amido (Fig. 5Q e 5R) no tricoma da nervura central, no parênquima medular do caule e na raiz. Compostos fenólicos (Fig. 5S) estão presentes no parênquima de preenchimento e na epiderme da nervura central, no parênquima de preenchimento e colênquima angular do pecíolo nos feixes vasculares do caule. Detectamos lipídios totais somente no parênquima do mesofilo (Fig. 5T). Mucilagens (Figs. 5U e 5V) estão presentes na nervura central, no pecíolo e no caule e evidenciamos óleos essenciais (Figs. 5X e 5Y) nos tricomas da nervura central, no parênquima de preenchimento do pecíolo e do caule.

As três espécies em estudo apresentam compostos antioxidantes, dentre os quais se destacam os compostos fenólicos, que também é encontrado em outra espécie da família Lamiaceae como Origanum vulgare L. (GONÇALVES et al., 2015) e Plectranthus barbatus (SILVA et al., 2016). Os compostos fenólicos se 
destacam por suas propriedades antioxidantes, anti-inflamatórias, protetora de órgãos vitais, incluindo o sistema cardiovascular (HITZ et al., 2018). O. basilicum possui elevado poder antioxidante, propriedade atribuída aos compostos fenólicos acumulados na forma de ácidos fenólicos (PITARO et al., 2012).

Assim como os demais compostos encontrados, as mucilagens também apresentam características terapêuticas, das quais podemos citar, atividades emolientes, proteção das inflamações cutâneas, desagregantes nas formulações de comprimidos, e também são utilizadas como antídoto nas intoxicações pelo iodo além de permitem a produção de xaropes com propriedades expectorantes (MOTA et al., 2014; DUTRA et al., 2016).

As propriedades medicinais atribuídas a óleos essenciais presentes nas espécies de Ocimum fazem com que sejam empregadas como anestésico, no tratamento de gripe, resfriados, e infecções respiratórias, ainda possui potencial inseticida, nematicida e antimicrobiano (ALMEIDA, 2011; OGENDO et al., 2008; LEMOS et al., 2005). Outra propriedade terapêutica está associada ao efeito antidepressivo, como revelado por uma pesquisa realizada em camundongos por Ali et al. (2017).

Através dos testes fitoquímicos revelamos a presença de flavonoides em apenas um órgão para cada espécie, sendo na raiz de $O$. basilicum e na inflorescência de $O$. campechianum e $O$. gratissimum. A atribuição medicinal dada aos flavonoides está associada a ações antioxidantes, caso comprovado em uma pesquisa com extrato aquoso de Melissa officinalis, espécie de Lamiaceae, onde foi verificado que o seu consumo em forma de chá pode realmente ter atividades antioxidantes (ARAÚJO et al., 2017).

Os taninos se destacaram em todos os órgãos, exceto na inflorescência de $O$. basilicum e $O$. campechianum e na raiz de 0 . gratissimum. Os taninos, atuam em locais lesionados, formando uma película protetora, promovendo a cicatrização, tal substância também é encontrada em outras espécies da família como em Leonurus sibiricus (VALENTINI et al., 2017). Detectamos saponina na folha de $O$. basilicum e $O$. campechianum, no caule de $O$. campechianum e $O$. gratissimum e na raiz de $O$. campechianum. Tradicionalmente espécies contendo saponinas são utilizadas como expectorantes e diuréticas (FIUZA et al., 2010). Em todos os órgãos estudados, verificamos a presença de alcalóides, exceto na inflorescência de $O$. basilicum, no caule e raiz de $O$. campechianum. Os alcalóides são compostos com uma estrutura diversificada e apresentam um amplo uso na farmacopeia, destacando sua atividade anestésica, antitumoral, miorrelaxante e antimicrobiológica (ALCANTARA et al., 2018).

\section{CONCLUSÕES}

A partir dos resultados obtidos podemos concluir que as características anatômicas observadas nas espécies $O$. basilicum e $O$. gratissimum são similares, enquanto a diferença de $O$. campechianum se dá pelo número de feixes vasculares e estrutura de raiz.

Os testes histoquímicos mostraram que as três espécies possuem similaridade nos compostos produzidos e os mesmos indicam potencial medicinal com ação analgésica e anti-inflamatória. Da mesma maneira, através dos testes fitoquímicos, podemos sugerir que as espécies possuem capacidade fitoterápica uma vez que os compostos nelas encontrados possuem relação com substâncias antioxidantes, 
expectorantes, anestésicas e antimicrobiana.

Os resultados desse estudo fornecem importantes informações quanto a localização dos compostos secundários para as espécies e podem subsidiar os estudos posteriores necessários para a produção de fitoterápicos, com maior ocorrência nos tecidos na epiderme, colênquima e parênquima de preenchimento.

Apesar das três espécies serem conhecidas pelo mesmo nome empírico (Alfavaca) a utilização das mesmas como tratamento alternativo estão sujeitas às respostas terapêuticas distintas e possíveis efeitos colaterais. Mostramos nesse trabalho que as espécies sintetizam os mesmos compostos secundários, no entanto, resta sabermos a composição destes que normalmente apresentam complexidade. As plantas medicinais devem ser utilizadas com muito critério, é necessário conhecer a planta e suas partes (raiz, caule, folhas e flores) que deverão ser usadas para liberar o princípio ativo.

AGRADECIMENTOS: Ao Conselho Nacional de Pesquisa (CNPq) e a Coordenação de Aperfeiçoamento de Pessoal de Nível Superior (CAPES) pelo suporte financeiro, a Fundação de Amparo à Pesquisa do Estado de Mato Grosso - FAPEMAT e ao Conselho Nacional de Desenvolvimento Científico e Tecnológico - CNPq (no 003/2016 - Programa de Desenvolvimento Científico e Tecnológico Regional - DCR).

\section{REFERÊNCIAS}

ALCANTARA, F. D. O.; SILVA, T. I.; MACIEL, T. C. M.; MARCO, C. A.; SILVA, F. B.. Teor e fitoquímica de óleo essencial de manjericão em diferentes horários de colheita. Journal of Neotropical Agriculture, v.5, n.4, p.1-6, 2018.

ALI, S. S.; ABD EL WAHAB, M. G.; AYUOB, N. N.; SULIAMAN, M.. The antidepressant-like effect of Ocimum basilicum in an animal model of depression. Biotechnic \& Histochemistry, v.92, n.6, p.390-401, 2017. DOI:

https://doi.org/10.1080/10520295.2017.1323276

ALMEIDA, M. A. Z.. Plantas Medicinais. 3 ed. Salvador: EDUFBA. 2011.

ARAÚJO, Í. P. C.; BATISTA, M. R. F.; SANTOS, T. B. A.; FERNANDO, W. L.; AMARAL, F. R.. Determinação da atividade antioxidante e teor de flavonoides totais equivalentes em quercetina em folhas de Cymbopogon citratus (dc) stapf e Melissa officinalis lam. Conexão Ciência, v.12, n.1, p.46-53, 2017. DOI:

https://doi.org/10.24862/cco.v12i1.499

CANINI, G. B.. Caracterização anatômica e composição química do óleo essencial de manjericão (Ocimum spp.). Dissertação (Mestrado em Agronomia) - Universidade de Brasília, Brasília, 2013.

COSTA, V. B. S.; CORRÊA, P. G.; DUTRA, M.; DAS CHAGAS, M. D. G. S.; DE MENDONÇA PIMENTEL, R.. Anatomia da folha e do caule de Ocimum gratissimum L. (Lamiaceae) sob condições naturais. Farmacognosia, v.17, p.549-556, 2014.

DAVID, R.; CARDE, R. J. P.. Coloration différentielle des inclusions lipidiques et terpéniques des pseudophylles du Pin maritime au moyen du réactif nadi. Paris: Comptes Rendus de l'Académie des Sciences. Serie lii-Sciencesde lavie-life Sciences, v.258, p.1338-1340, 1964.

DORES, R. G. R.. Histoquímica de Dimorphandra mollis Benth. Dissertação (Mestrado em Agronomia) Universidade Federal de Viçosa, Viçosa, 2007.

DUARTE, M. R.; LOPES, J. F.. Morfoanatomia foliar e caulinar de Leonurus sibiricus L., Lamiaceae. Acta Farmacéutica Bonaerense, v.24, n.1, p.68-74, 2005.

DUARTE, M. R.; LOPES, J. F.. Stem and leaf anatomy of Plectranthus neochilus Schltr., Lamiaceae. Revista Brasileira de Farmacognosia, v.17, n.4, p.549-556, 2007. DOI: http://dx.doi.org/10.1590/S0102-695X2007000400013

DUTRA, R. L.; CRIVELLI, S. R. M.; FRITZEN, M.. Farmacognosia I. Estácio, 2016.

ESTEVES, L. M.. Meio ambiente e botânica. São Paulo: SENAC, 2011.

FERNANDES, F. F.. Crescimento, produção do óleo essencial e anatomia foliar de Ocimum gratissimum L. (lamiaceae) em diferentes níveis de radiação luminosa. Dissertação (Mestrado em Produção Vegetal) - Universidade Estadual de Santa Cruz, Ilhéus, 2012.

FIUZA, T. S.; REZENDE, M. H.; SABÓIA-MORAIS, S. M.; TRESVENZOL, L. M.; FERREIRA, H. D.; PAULA, J. R.. Estudo das folhas e caule de Hyptidendron canum (Pohl ex Benth.) Harley, Lamiaceae. Revista Brasileira de Farmacognosia, v.20, n.2, p.192-200, 2010. 
FURLAN, M. R.; AOYAMA, E. M.; INDRIUNAS, A.; MAURO, C.. Anatomia Foliar de Ocimum basilicum L. Genovese (Lamiaceae). Revista Fitos, v.7, n.4, p.231-235, 2012.

GANTER, P.; JOLLÉS, G.. Histologie normale et pathologique. Paris: Gauthier Villars, 1970.

GEISSMAN, T. A.; GRIFFIN, T. S.. Sesquiterpene lactones: Acid-catalyzed color reactions as an in structure determination. Phytochemistry, v.10, n.10, p.2475-2485, 1971. DOI: https://doi.org/10.1016/S0031-9422(00)89894-5

GONÇALVES, J. H. T.; SANTOS, A. S.; MORAIS, H. A.. Atividade antioxidante, compostos fenólicos totais e triagem fitoquímica de ervas condimentares desidratadas. Revista da Universidade Vale do Rio Verde, v.13, n.1, p.486-497, 2015. DOI: http://dx.doi.org/10.5892/ruvrd.v13i1.2003

HARDMAN, R.; SOFOWORA, E. A.. Antimony trichloride as test reagents for steroids, especially diosgenin and yamogenin, in plant tissues. Stain Technology, v.47, p.205208, 1972. DOI:

https://doi.org/10.3109/10520297209116486

HARLEY, R.; FRANÇA, F.; SANTOS, E. P.; SANTOS, J. S.; PASTORE, J. F.. Lamiaceae. In: Lista de Espécies da Flora do Brasil. Rio de Janeiro: Jardim Botânico do Rio de Janeiro, 2015.

HITZ, D.; BARBOSA, M.; NEZELLO, M. C.; MAZUR, C. E.. Ação dos compostos fenólicos na aterosclerose: uma revisão. Visão Acadêmica, v.19, n.1, p.107-112, 2018. DOI: http://dx.doi.org/10.5380/acd.v19i1.57729

JOHANSEN, D. A.. Plant microtechnique. New York: MacGraw-Hill, 1940.

JORGE, S. S. A.. Plantas Medicinais: Coletânea de Saberes. 2013.

KRAUS, J. E.; ARDUIN, M.. Manual básico de métodos em morfologia vegetal. Rio de Janeiro: Universidade Rural, 1997.

LEMOS, J. D. A.; PASSOS, X. S.; FERNANDES, O. D. F. L.; PAULA, J. R. D.; FERRI, P. H.; LEMOS, A. D. A.; SILVA, M. D. R. R.. Antifungal activity from Ocimum gratissimum $L$. towards Cryptococcus neoformans. Memórias do Instituto Oswaldo Cruz, v.100, n.1, p.55-58, 2005.

MACE, M. E.; HOWELL, C. R.. Histochemistry and identification of condensed tannin precursors in roots of cotton seedlings. Canadian Journal of Botany, v.52, n.11, p.2423-2426, 1974. DOI: https://doi.org/10.1139/b74-314

MAECHLER, M.; ROUSSEEUW, P.; STRUYF, A.; HUBERT, M.; HORNIK, K.. Cluster: Cluster Analysis Basics and Extensions. R package version 2.0. 2015.

MATOS, F. J. A.. Introdução à Fitoquímica Experimental. Fortaleza: UFC, 2009.

METCALFE, C. R.; CHALK, L.. Anatomy of Dicotyledons. 2 ed. Oxford: Oxford University Press, 1979.

MOTA, T. H. S.; SOUZA, S. R.; SANTOS, A. P.; CUNHA, C. R. M.. Estudo farmacognóstico das folhas da Sterculia striata
St. Hil. Et. Naid., coletadas em Itapuranga-Go. Revista Eletrônica Faculdade Montes Belos, v.7, n.1, p.34-68, 2014.

OGENDO, J. O.; KOSTYUKOVSKY, M.; RAVID, U.; MATASYOH, J. C.; DENG, A. L.; OMOLO, E. O.. Bioactivity of Ocimum gratissimum $\mathrm{L}$. oil and two of its constituents against five insect pests attacking stored food products. Journal of Stored Products Research, v.44, n.4, p.328-334, 2008. DOI: https://doi.org/10.1016/j.jspr.2008.02.009

OKSANEN, J.; BLANCHET, F. G.; KINDT, R.; LEGENDRE, P.; MINCHIN, P. R.; O'HARA, R. B.; OKSANEN, M. J.. Vegan: Community ecology package. R package version 2.0. 2013.

OLIVEIRA, A. B. D.. Estudo estrutural e farmacognóstico de Scutellaria agrestis A. St.-Hil. ex Benth. (Lamiaceae): uma planta medicinal utilizada por populações ribeirinhas do Amazonas. Tese (Doutorado em Botânica) - Instituto Nacional de Pesquisas da Amazônia, Manaus, 2011.

OLIVEIRA, A. B.; MENDONÇA, M. S.; MEIRA, R. M. S. A.. Anatomy of vegetative organs of Scutellaria agrestis, a medicinal plant cultivated by riverine populations of the Brazilian Amazon. Revista Brasileira de Farmacognosia, v.23, n.3, p.386-397, 2013. DOI: http://dx.doi.org/10.1590/S0102-695X2013005000034

PEARCE, J. A.; GALE, G. H.. Geochemical evidence for the genesis and eruptive setting of lavas from Tethyan ophiolites. In: PANAYIOTOU, A.. Ophiolites: Proceeding International Ophiolite Symposium. Cyprus, 1980. p.261272 .

PITARO, S. P.; FIORANI, L. V.; JORGE, N.. Potencial antioxidante dos extratos de manjericão (Ocimum basilicumLamiaceae) e orégano (Origanum vulgare- Lamiaceae) em óleo de soja. Revista Brasileira de Plantas Medicinais, v.14, n.4, p.686-691, 2012. DOI: http://dx.doi.org/10.1590/S1516$\underline{05722012000400017}$

PIZZOLATO, T. D.; LILLIE, R. D.. Mayer's tannic acid-ferric chloride stain for mucins. The Journal of Histochemistry and Cytochemistry, v.21, p.56-64, 1973. DOI:

https://doi.org/10.1177/21.1.56

RIVA, A. D.; PETRY, C.; SEVERO, B. M. A.. Caracterização anatômica de folhas e inflorescências de espécies de Lavanda (Lamiaceae) utilizadas como medicinais no Brasil. Ciência e Natura, v.36, n.2, p.120-127, 2014. DOI: https://doi.org/10.5902/2179460X13654

ROCHA, A. M.; PASSADOR, A. L.; MAIA, R. V.; PAULINO, I. L. S.; SILVA, I. V.. Ocimum basilicum L. (Lamiaceae): Uso potencial em aulas práticas de Anatomia Vegetal. In: SEMINÁRIO DE BIODIVERSIDADE E AGROECOSSISTEMAS AMAZÔNICOS, 3. Anais. Cáceres: UNEMAT, 2015. p.297-302.

ROESER, K. R.. Die Nadel der Schwarzkiefer Massenprodukt und Kunstwerk der Natur. Mikrokosmos, v.61, p.33-36, 1962.

SILVA, C. F. G.; MENDES, M. P.; ALMEIDA, V. V.; MICHELS, R. N.; SAKANAKA, L. S.; TONIN, L. T. D.. Parâmetros de qualidade físico-químicos e avaliação da atividade antioxidante de folhas de Plectranthus barbatus Andr. (Lamiaceae) submetidas a diferentes processos de secagem. Revista Brasileira Plantas Medicinais, Campinas, v.18, n.1, 
p.48-56, 2016. DOI: http://dx.doi.org/10.1590/1983$084 \mathrm{X} / 15021$

SILVA, R. M.; FARIA, M. T.. Caracterização etnobotânica e histoquímica de plantas medicinais utilizadas pelos moradores do Bairro Carrilho, Goianésia - GO. Enciclopédia Biosfera, v.10, n.19, p.23, 2014.

SIMON, E. H.; ROJAS, M. C. C.; LE VRAUX, M. A.; GONZÁLEZ, F. S.. Estudio anatómico de órganos vegetativos de dos especies de Ocimun L. (Lamiaceae); Vegetative anatomy study in two species of Ocimun L. (Lamiaceae). In: JORNADAS
ARGENTINAS DE BOTÁNICA, 33. Anais. Buenos Aires: Boletín de la Sociedad Argentina de Botánica, 2011. p.120-121.

STEVENS, P. F.. Angiosperm Phylogeny Website. Version 14, July 2017. 2018.

SVENDSEN, B. A.; VERPOORTE, R.. Chromatography of alkaloids. New York: Elsevier Scientific Publish Company, 1983.

VALENTINI, S. A.; CASALE, F. M.. Controle de qualidade de formas farmacêuticas tópicas utilizando diferentes extratos vegetais. Revista Iniciare, v.2, n.1, 2017.

A CBPC - Companhia Brasileira de Produção Científica (CNPJ: 11.221.422/0001-03) detém os direitos materiais desta publicação. Os direitos referem-se à publicação do trabalho em qualquer parte do mundo, incluindo os direitos às renovações, expansões e disseminações da contribuição, bem como outros direitos subsidiários. Todos os trabalhos publicados eletronicamente poderão posteriormente ser publicados em coletâneas impressas sob coordenação da Sustenere Publishing, da Companhia Brasileira de Produção Científica e seus parceiros autorizados. Os (as) autores (as) preservam os direitos autorais, mas não têm permissão para a publicação da contribuição em outro meio, impresso ou digital, em português ou em tradução. 